Journal of Social Sciences 6 (2): 276-281, 2010

ISSN 1549-3652

(C) 2010 Science Publications

\title{
Grounded Theory: A Short Cut to Highlight a Researchers' Intellectuality
}

\author{
${ }^{1}$ Abdul Hafidz Haji Omar, ${ }^{2}$ Dayang Hjh. Tiawa Awang Hj. Hamid, \\ ${ }^{3}$ Norma Alias and ${ }^{4} \mathrm{Md}$. Rajibul Islam \\ ${ }^{1}$ Department of Therapy and Rehabilitation, Faculty of Biomedical and Health Science Engineering, \\ ${ }^{2}$ Department of Multimedia Education, Faculty of Education, \\ ${ }^{3}$ Ibnu Sina Institute, Faculty of Science, \\ University Technology Malaysia, Malaysia \\ ${ }^{4}$ Faculty of Information Science and Technology, Multimedia University, Malaysia
}

\begin{abstract}
Problem statement: Grounded theory is one of the research approaches in qualitative research that enables the researcher to use his/her intellectuality to make sense of and construct a theory based on finding collected throughout the fieldwork. Grounded Theory as suggested in the conventional studies is designated in a very comprehensive manner and involves three level of coding. These coding systems are parallel to Bloom's Taxonomy where an individual has to first understand the basic knowledge before he/she is able to proceed further. Approach: In the first stage of Grounded Theory, that is, 'open coding'; data are grouped according to themes or categories. That is where the researcher has to have sufficient knowledge and understanding of the phenomenon being studied. In the second level, that is, 'axial coding'; the researcher has to connect the themes and make sense of it. At this point, the researcher applies his knowledge and analyses the data. The data is then synthesized and evaluated through 'selective coding'. Results: These will develop into the draft of the theory that will be tested against other existing theories to form 'Grounded Theory'. Conclusion: This study showed that the ability to develop a good 'Grounded theory' will represent the researchers' intellectuality.
\end{abstract}

Key words: Grounded theory, Bloom's taxonomy, researchers, intellectuality

\section{INTRODUCTION}

This study is divided into two parts. Part I discusses the theoretical aspects of Grounded Theory while Part II looks at putting theory into practice.

Part I: Theoretical aspects of grounded theory: Grounded Theory as a method used in qualitative research was first introduced by Glaser and Strauss (1967). This method was later updated by Glaser (1978) and improved on by Strauss (1987). Terms closely associated with this method are interviews, building concepts, links/relationships between data and other terms which connote the inductive process of data analysis. According to Strauss and Corbin (1990), Grounded Theory is qualitative research method where a researcher "derive[s] inductively from the study of a phenomenon it represents". The term grounded theory can better be described as:
"A grounded theory, is one that is inductively derived from the study of the phenomenon it represents. That is, discovered, developed and provisionally verified through systematic data collection and analysis and theory should stand in reciprocal relationship with each other. One does not begin with a theory and then prove it. Rather, one being with an area of study and what is relevant to that area of study and what is relevant to that area is allowed to emerge" (Strauss and Corbin, 1990)

Pandit (1996) identified three basic elements needed in developing a grounded theory: Concept building, data classification or categorization and stating the phenomenon. Concepts are basic units derived from data gathered in the study. However, Strauss and Corbin (1990) rank categories higher than concepts, as this is where the researcher begins to look at the similarities and differences that exist at the

Corresponding Author: Abdul Hafidz Haji Omar, Department of Therapy and Rehabilitation, Faculty of Biomedical and Health Science Engineering, University Technology Malaysia, Malaysia Tel: +60197256109 
concept level. Linking or looking for relationships that exist between the concepts found in the category result in the phenomenon.

The main aim of Grounded Theory is to build a theory based on naturalistic/real data. This being with the researcher investigating and trying to get an indepth understanding of a particular phenomenon by looking at the similarities and differences that exist in the data collected in the field. In analyzing data, the researcher beings at the micro level and moves toward the micro level and at the micro level the researcher analyzes the data and tries to look for links that exist within the data and hypothesizes its relationship with the phenomenon. This is similar to Newman (2002) positive-oriented theory -- a theory should have data that can support, strengthen a phenomenon so that it can be tested, replicated and generalized.

In building a grounded theory, the term coding is used to look for relationships between categories. Strauss and Corbin (1990) describe the coding process as:

"Analysis or coding of qualitative data represents the operations by which data are broken down, conceptualized and put back together in new ways. It is the central process by which theory is built from data"

In the coding process, data is sorted and analyzed inductively using the three-stage model of open coding, axial coding and selective coding. Open coding involves identifying concepts based on certain criteria. Data gathered from interviews, documents, observations and other sources are compared and sorted according to themes. This involves the process of categorization before moving on to the second stage i.e., axial coding. In this stage, the researcher begins to look for link/relationships between categories and subcategories. This is done through interviews and also observations that focus primarily on information which are linked to the related themes. The story line is developed through the comparative comparison method that supports and validates the relationships and establishes a story line that describes the phenomenon. In this way, subsequent data collection efforts will look for answers to any new questions or concerns that emerge from the previous analysis. In the selective coding stage, the researcher looks for recurring regularities in the categories. This is then organized into key patterns or themes.

Throughout the coding process, the researcher is constantly aware of the developments in the data being analyzed and validating them with new themes that emerge (Kelle, 1995). Data collection ceases when the data is saturated. Data can become saturated in two ways: Theoretical saturation and sampling saturation. Theoretical saturation occurs when the data being analyzed shows recurring regularities, while sampling saturation occurs when all the respondents are consistently providing the same type of information. This is an indicator that the analysis is almost at the final stage. The findings at this stage are compared to previous findings, allowing room for discussion. The researcher is also beginning to come up with draft theory/theories that will then be tested against existing theories to build a Grounded Theory.

This study attempts to show the relationship between Grounded Theory and Bloom's Taxonomy. The writers feel that there are similarities in the processes involved in building a Grounded Theory and Bloom's Taxonomy, which discusses the hierarchy, involved when an individual develops his/her cognitive competencies. Benjamin introduced the Taxonomy in (Bloom, 1956). The thrust of the Taxonomy is the classification of an individual's cognitive behavior in learning. This hierarchical classification of the Taxonomy is divided into six levels: Knowledge, comprehension, application, analysis, synthesis and evaluation. Bloom (1956) explains:

Taxonomy simply means classification. So the well-known objectives is an attempt (within the behavioral paradigm) to classify forms of learning. It is suggested that one cannot effectively or ought not to try to address higher levels until those below them have been covered (it is thus serial in structure). It also suggests a way of categorizing levels of learning.

The lowest level within Bloom's Taxonomy is knowledge. At this level, an individual observes or tries to recall what is learned. The behavioral objectives at this level require an individual to list, label and state what they have learned through the recall process.

At the comprehension level of Bloom's Taxonomy, an individual should be able to explain what is learned, observed or heard. The individual is also expected to summarize data, identify similarities or differences and should have the ability to discuss the data. At the application level, i.e., the third level of Bloom's Taxonomy, an individual utilizes the data to solve problems. This requires an individual's ability to think critically. In relating this to Grounded Theory, the researcher is expected to sort, build concepts and classify data according to themes based on similarities and/or differences (Lee and Fielding, 1991).

At the fourth level of Bloom's Taxonomyanalysis, the researcher should analyze the data by looking at the similarities and/or differences, look for links within the data, look for possible story lines. At 
the fifth level of Bloom's Taxonomy, the researcher synthesizes the data, which has been analyzed with new information. He/she then generalizes the information, reorganizes the information and presents it in a different form.

The evaluation level of Bloom's Taxonomy, the researcher evaluates, makes decisions, explains new findings and tests/validates his/her work against the study of others and makes informed choices.

Many similarities exist between Bloom's Taxonomy and Grounded Theory. The most interesting feature of Grounded Theory is that it places the researcher at the highest level of Boom's Taxonomy. Through this, the researcher analyzes his findings and eventually comes up with draft theories or new theories. This feature is not exemplified when a researcher uses other research methods such as survey or case studies.

The coding process of Grounded Theory reflects how a researcher begins to comprehend or understand data obtained from the fieldwork. This fits in with Levels 1 and 2 of Bloom's Taxonomy where the learner begins to understand and classify the data according to themes based on their similarities and/or differences. At the axial coding stage, the researcher analyzes the data based on his understanding of the data. This parallels Levels 3 and 4 of Bloom's Taxonomy where relationships between data i.e., the categories are identified to explain what is actually happening in the field. At Level 5 and 6 of Bloom's Taxonomy, the researcher's ability to data occurs. A similar process occurs at the selective coding stage of Grounded Theory where the main themes begin to be formulated to explain the findings (Richards and Richards, 1994). This stage also oversees the building of draft theories where the researcher validates his/her findings against the study of others or other existing theories.

The Grounded Theory method can be considered as comprehensive based on the following:

- The enormous amount of data collected before links/relationships between the categories can be done with accuracy

- Data saturation will not be reached if the data being analyzed is insufficient or does not show recurring regularities

- To obtain an accurate outcome.

- A combination of both qualitative and quantitative methods can produce a

- higher level of validity and reliability Use of triangulation where data obtained through different methods are tested against each other

To get feedback on his findings, a researcher is encouraged to present his findings at seminars or through journal publications. This provides the researcher with the opportunity of being biased. The feedback obtained can assist the researcher to review his findings and eventually look into ways to increase the validity and reliability of his findings (Burns, 1994). This provides an avenue for the researcher to publicize his findings, which were obtained through an organized and comprehensive method of data collection.

Indirectly, the researcher has taken a shortcut to evaluate his intellectuality. If his/her findings are accepted, it is an acknowledgment of his/her intellectuality and can open avenues for more research. On the other hand, if weaknesses exist in the findings, the researcher has the flexibility of altering the boundaries that had been set for the study and make the necessary adjustments depending upon what happens in the field. Doing so, allows the researcher to provide a thick description of the study as it occurs it its natural setting.

Part II: Putting theory into practice: This part demonstrates the relationship between that exists between Grounded Theory and Bloom's Taxonomy in a study that was done by one of the writers. Details of the study are listed in Table 1:

- Title of study: The Influence of Culture on Elite National Malaysian (Malay-Muslims) athletes

- Sample: About 32 elite national Malaysian (MalayMuslims) athletes representing various sports

- The objective of this study is to develop the Grounded Theory on the influence of culture on elite National Malaysian (Malay-Muslims) athletes.

\section{MATERIAL AND METHODS}

The study is designed according to the procedure developed by Strauss and Corbin (1990). In the initial stage, 'open coding' was used as an analytical process which identified and developed themes in term of their properties and dimensions. Later the researcher develops, specifies and manages multiple linkages between text segments, between text segments and codes and between codes themselves using 'axial coding'. As the analysis progresses, the researcher using constant comparison, compares all pieces of data with other pieces and identifies the core variables. These core variables are the central variables and are tested to many other categories to form core categories (Glaser, 1978). These categories were tested across the full range of categories both new and old using 'selective coding' until it was clear that no fresh code were about to emerge. This is the stage where the researcher is able to construct draft theory. 
J. Social Sci., 6 (2): 276-281, 2010

Table 1: Initial categories (open coding)

\begin{tabular}{|c|c|c|}
\hline \multirow[b]{2}{*}{ Different cultural influences } & \multicolumn{2}{|c|}{ Malay-Muslim society elite athletes' background } \\
\hline & Male & Female \\
\hline Islam & Boxing & Netball \\
\hline Hindu-Buddhist & Badminton & Archery \\
\hline Malay Traditional & Rugby & Artistic gymnastic \\
\hline Post Colonial & Weight lifting & Cycling \\
\hline \multirow[t]{2}{*}{ Animism } & Athletic & Hockey \\
\hline & Hockey & Swimming \\
\hline \multicolumn{3}{|l|}{ Athletes' daily practices in sport } \\
\hline Reasons for sport involvement & Religious obligations & \\
\hline National pride & Daily prayers & \\
\hline Money or reward & Fasting & \\
\hline Family Pride & Free mixing & \\
\hline Fitness & Status of Muslim sportswomen & \\
\hline Love of sport & Married Muslim sportswomen & \\
\hline Desire to be champion & Dietary aspects & \\
\hline Sports' etiquette & Positive attitudes & \\
\hline Rule of the game & Good health & \\
\hline Mixed events & Good personality & \\
\hline Games attire & Raise socio-economic status & \\
\hline Interaction with coaches & Social harmony & \\
\hline Win at all costs & Customs and traditions & \\
\hline \multicolumn{3}{|l|}{ Fair play } \\
\hline \multicolumn{3}{|l|}{ Friendly match } \\
\hline \multicolumn{3}{|l|}{ Negative behaviors } \\
\hline Betting & Dangerous sports & Immoral; advertisements \\
\hline Corruption & Contact sport & \\
\hline Drug usage gambling & Aggression & \\
\hline
\end{tabular}

The researcher then triangulated the core categories constructed from different data collecting methods, sources, investigators and finally referred to relating theories and literature. Richards and Richards (1994) noted that testing and rechecking the relationship between categories, core categories and draft theories, enables the researcher to achieve two objectives: (a) theoretical sampling and (b) theoretical saturation, that will determine the point at which to stop the data collection. The final outcomes led to the formation of the grounded theories of this study.

\section{RESULTA AND DISCUSSION}

For this study, in the open coding stage, the initial categories are equivalent to the knowledge and comprehension levels of Bloom's Taxonomy. Here the researcher seeks to understand the data and break them into categories based on similarities and differences in themes using the constant comparative method. Example of the categories developed in the study is shown in Table 1.

The data in this study was analyzed using the Non Unstructured Data Indexing Searching Theorizing (NUDIST) computer software package (Tesch, 1990) where the themes were organized into a Tree Index System as shown in the Fig. 1 .
In the axial coding stage, the researcher looks for relationships between the categories. A similar process occurs during the application and analysis levels of Bloom's Taxonomy that requires the researcher to establish the relationships that exist between information accumulated in the past to newly gained information. A comprehensive data analysis is done at this stage, how the researcher was able to conduct the process and retrieve all the information regarding a Muslim female swimmer describing her dilemma about whether to swim or fast. In actual fact it is impossible to swim and fast. The second feature observed to have strong influence on the athletes' practices in sports in the different between the practices of a sub culture (colonial culture) in sports and the dominant culture (Muslim Culture) of a society. This study found that several elements in the sub culture contradict with the value of the dominant culture Fig. 2.

In the selective coding stage, the main themes are formulated as the researcher undergoes the synthesis process of Bloom's Taxonomy i.e., the fifth level of the hierarchy. This leads to the final stage of Grounded Theory, i.e., building a draft theory. A similar process occurs at the highest level of Bloom Taxonomy's where the researcher begins to evaluate the draft theories. The final outcome is called Grounded Theory-a stage in which the researcher is able to evaluate, make conclusions and explain findings of the study or research. 


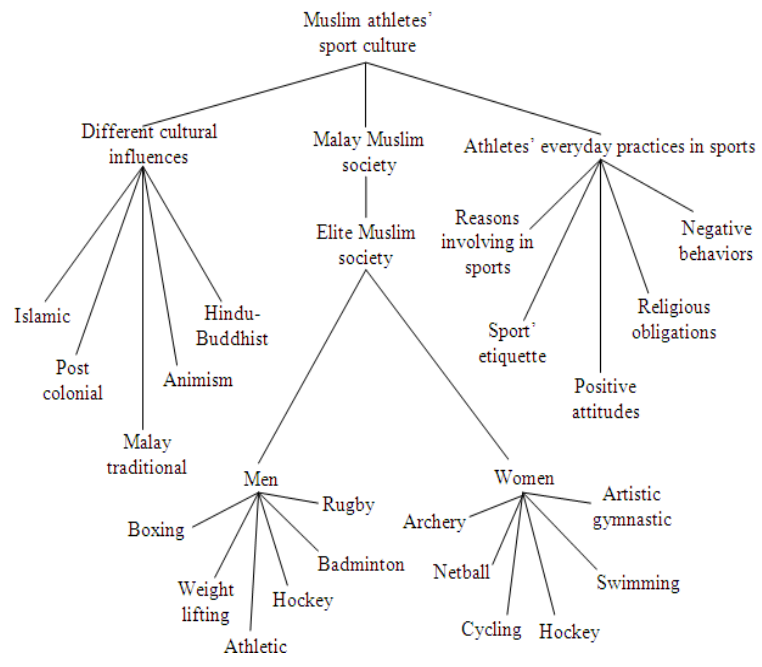

Fig. 1: Initial coding/open coding, tree index system of Malaysian Muslim athletes' sport culture

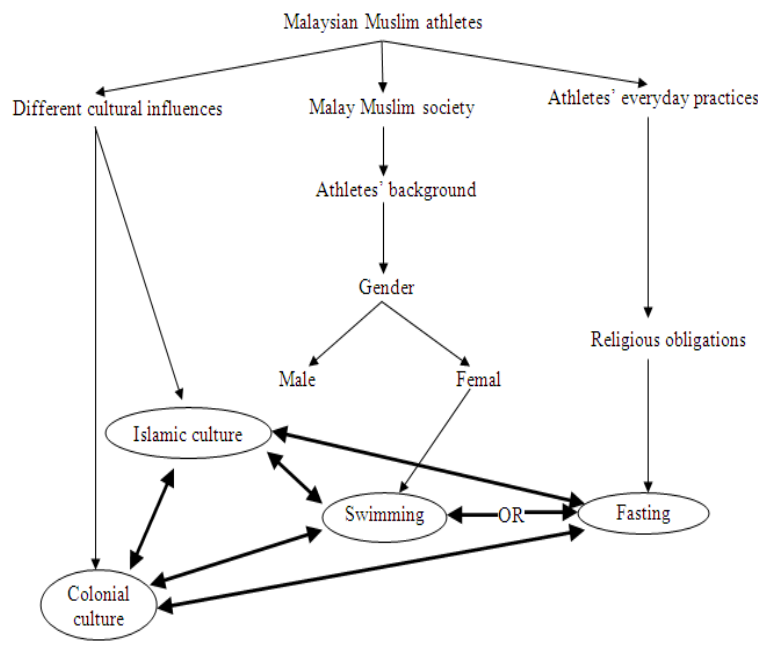

Fig. 2: Comparison between Islamic and colonial culture regarding fasting

The theory building process of this study is illustrated in the Fig. 3.

This study also came up with several draft theories such as:

- The different levels of an individual's faith had a great influence on the athletes' ability to practice their Islamic culture in sports

- The difference between values in the sub-culture (everyday practices in sport) and the dominant culture (Islamic culture as the norms of the Malay society) plays an important role in the athletes' practices and has an impact on their sports behavior

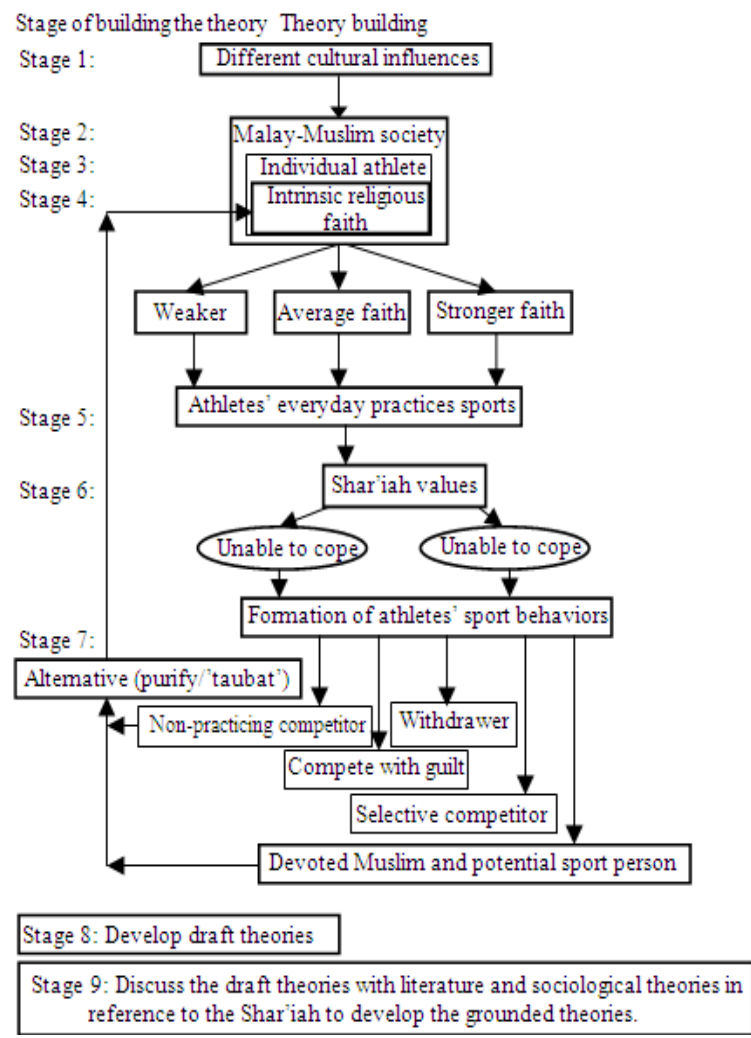

Fig. 3: The flowchart of building proposed theory

- Experienced athletes are able to adapt themselves and follow the Shar'iah better than the less experienced athletes

- Muslim Sports women faced more difficulties than men did in elite sports

The draft theories were then tested and debated against existing theories and literatures to build a grounded theory. The grounded theories for this study are:

- To Muslim athletes whose religious faith plays an important role in deciding the sport behaviors which develop in sport culture

- To develop a strong faith, Muslims must keep away from all negative acts in sport; create a healthy culture which will in turn develop a healthy society

- In a Muslim society, culture of sport develops healthy individuals, unites the community and prevents hatred among mankind

- Muslim sports women are obliged to be with their gender when involved in sport and competition. They are also required to choose those sports that reflect their feminine values in other to guard their honor and dignity 


\section{CONCLUSION}

A researchers' ability to develop a Grounded Theory in qualitative research illustrates the highest level of intellectuality in term of Bloom's Taxonomy. Publication of the research results indirectly manifests the researcher's intellectual ability. This study encourages researchers to utilize their areas of analysis, to suggest criteria to estimate the significance of discovered theory, to recommend techniques for doing so.

\section{REFERENCES}

Bloom, B.S., 1956. Taxonomy of Educational Objectives, Handbook I: The Cognitive Domain. 2nd Edn., Wesley Publishing Company, ISBN: 13: 978-0582280106, pp: 113.

Burns, R.B., 1994. Introduction to Research Methods. 2nd Edn., Longman Cheshire, Melbourne, ISBN: 0582911877, pp: 436.

Glaser, B.G., 1978. Theoretical Sensitivity: Advances in the Methodology of Grounded Theory. Sociology Press, Mill Valley, CA., ISBN: 13: 978-1884156014, pp: 164.

Glaser, B. and A. Strauss, 1967. The Discovery of Grounded Theory: Strategies for Qualitative Research. Aldine Transaction, New York, ISBN: 13: 978-0202302607, pp: 271.

Kelle, U., 1995. Computer-Aided Qualitative Data Analysis: Theory, Methods and Practice. Sage Publications Ltd., London, ISBN: 13: 9780803977617, pp: 224.
Lee, R.M. and N. Fielding, 1991. Computing in Qualitative Research: Options, Problems and Potential. In: Using Computers in Qualitative Research, Fielding, N. and R. Lee (Eds.). SAGE Publications, London, ISBN: 13: 9780803984257.

Newman, W.L., 2002. Social Research Methods: Qualitative and Quantitative Approaches. 5th Edn., Allyn and Bacon, Needham Heights, Massachusettes, ISBN: 13: 978-0205353118, pp: 592.

Pandit, N.R., 1996. The creation of theory: A recent application of grounded theory method. Q. Rep., 2: 1-14. http://www.nova.edu/ssss/QR/QR24/pandit.html

Richards, T. and L. Richards, 1994. From Filing Cabinet to Computer. In: Analyzing Qualitative Data. Burgess, R.W. and Bruman (Eds.), Routledge, UK., ISBN: 978-0-415-06063-9.

Strauss, A.L. and J. Corbin, 1990. Basic of Qualitative Research: Grounded Theory Procedures and Techniques. 2nd Edn., SAGE Publications, London, ISBN: 13: 978-0803932517, pp: 272.

Strauss, A.L., 1987. Qualitative Analysis for Social Scientists. Cambridge University Press, Cambridge, ISBN: 13: 9780521338066, pp: 336.

Tesch, R., 1990. Qualitative Research: Analysis Types and Software. 1st Edn., Routledge, UK., ISBN: 13: 978-1850006091, pp: 344. 